\title{
Implementación de un sistema de gestión de la calidad para mejoras en la empresa
}

\author{
Mario Mauricio Herrera \\ Corporación Allflex Inc. Québec, Canadá \\ Correo electrónico: MMauricio@allflexusa.com
}

Recibido: 14/11/2011 / Aprobado: 19/1/2012

\begin{abstract}
Resumen: La empresa A Canadá, compañía dedicada a la producción y comercialización de identificadores de activos, hizo frente al desafío de continuar con la reorganización de sus actividades al mismo tiempo que implementaba la norma ISO 9001. Un cuidadoso análisis preliminar y la adaptación de las herramientas y exigencias de la norma para su caso en particular resultaron en la mejora del tiempo de respuesta y nivel de servicio para sus clientes. Posteriormente, el nivel de producción aumentó considerablemente al trasladarse de vuelta la producción hecha por otras plantas del grupo.
\end{abstract}

Palabras clave: ISO 9001 / mejora continua / gestión de la calidad / control de procesos

\section{Implementation of a quality management system for improvement}

\begin{abstract}
Company A Canada, dedicated to the production and commercialization of assets tracking devices, faced the challenge of keeping the reorganization of its activities while implementing the ISO 9001 norm. A careful preliminary analysis and the use adapted for its own needs of the tools and requirements provided by the norm resulted in the improvement of the response time and level of service for its customers. Later on, its production level increased after other plants of the group transferred back the production for the Canadian market.
\end{abstract}

Keywords: ISO 9001 / continuous improvement / quality management/ process control 


\section{INTRODUCCIÓN}

\subsection{Necesidad de una respuesta predecible}

$\mathrm{Al}$ momento de adquirir un bien o contratar un servicio, el cliente, ya sea una persona natural o jurídica, espera poder llenar una necesidad con lo adquirido; visto así, puede decirse que la necesidad es el motor de la compra. Una vez hecha la adquisición, el cliente la evalúa: ¿este producto o servicio realmente responde a mi necesidad? Esta evaluación puede ser muy elaborada, basada en criterios y tolerancias preestablecidas, o ser muy vaga y basarse en la impresión general del bien o el servicio.

La respuesta a esta pregunta, en combinación con la presencia de la misma necesidad, llevará a repetir o no la misma compra.

$\mathrm{Al}$ analizar esta dinámica bastante simplificada de la compra-venta es posible ver una constante en ella: al momento de la compra el cliente actúa basado tanto en su necesidad como en la idea de que el producto o servicio es el adecuado para dicha necesidad. Esta idea puede ser generada por la publicidad del vendedor, por una experiencia previa, por recomendaciones de un tercero o por una combinación de estas tres razones; y es aquí donde la necesidad de una respuesta predecible se manifiesta.

Evidentemente, la fidelidad del cliente estará basada, entre otras cosas, en la misma respuesta del producto o servicio para su necesidad. Si su necesidad evoluciona, y se quiere seguir conservándolo como cliente, es necesario que la respuesta del producto o servicio evolucione en consecuencia. Hay quienes opinan que lo ideal es que se guíe la evolución de dicha necesidad en una dirección en la que sea más fácil diferenciarse de los competidores.

$\mathrm{Al}$ analizar este enunciado es posible ver la utilidad de ser capaces de ofrecer una respuesta predecible. Como es lógico, si no se tiene el control de los procesos internos, será imposible tener control sobre la respuesta del producto o servicio.

\subsection{Cómo puede ayudar un sistema de gestión de la calidad}

Un sistema de gestión de la calidad puede ayudar a tener un mejor control de cada uno de los procesos internos. Este control es una herra- 
mienta muy útil si se combina con la necesidad de ofrecer una respuesta predecible a los clientes.

Existen, naturalmente, algunas condiciones para que la ayuda del sistema de gestión de la calidad sea factible, y una de esas condiciones es que dicho sistema no sea simplemente una entidad basada en documentos y registros, sino que estos documentos y registros sean consecuencia del control que tenemos sobre nuestros procesos. Existe una gran diferencia entre estas dos implementaciones de un sistema de gestión de la calidad.

\section{EMPRESA A}

\subsection{La compañía}

La empresa A es una compañía dedicada a la identificación de activos, presente en Canadá, Estados Unidos, Europa, Australia, Nueva Zelanda y Brasil, entre otros países. ${ }^{1}$

La presencia de la empresa A en Canadá es la respuesta de la compañía — a inicios de la década del 2000 - a la implementación de programas de identificación de activos puesta en marcha por diferentes instancias del gobierno federal y de los gobiernos provinciales en Canadá.

\subsection{La situación}

Para el año 2005, el sistema de identificación de activos en el mercado canadiense se acercaba a los cinco años de experiencia, y la demanda por identificadores se acentuaba, pues cada vez era mayor el número de criadores que utilizaban el sistema. Sin embargo, la capacidad de la planta en Canadá no era suficiente para atender la demanda. Parte de la producción fue trasladada a las plantas en Europa y Estados Unidos, a pesar del aumento del costo de la logística de las operaciones.

A mediados del año 2005 es claro que debió haber un cambio en la gestión de la planta en Canadá para restablecer la cadena de abastecimiento de la empresa A.

1 Declaración de la política de calidad de la empresa A. Canadá (2010). 


\subsection{Cambios iniciales}

Un análisis rápido de la situación de la planta permitió determinar que había ineficiencias tanto en la programación de la producción como en la ejecución de dicha programación. Luego de este análisis se iniciaron los cambios en el núcleo de producción de la planta, con lo que se sentaron las bases para una posterior reorganización de la producción para el mercado canadiense.

\subsection{La necesidad de reorganizar el sistema actual versus la necesidad de obtener un certificado de sistema de gestión de la calidad}

Durante el año 2006 uno de los principales clientes de la empresa A expresó su deseo de obtener el Certificado ISO 9001 para sus actividades de identificación y trazabilidad de ganado. La dirección de la empresa A entendió que este cambio en uno de los principales agentes del mercado no pasaría desapercibido, y que era necesario responder adecuadamente.

Se tomó la decisión de implementar en la planta de Canadá un sistema de gestión de la calidad basado en la norma ISO 9001; sin embargo, esta misma planta aún estaba en pleno proceso de reorganización y ese proceso utilizaba ya muchos de los recursos de la empresa.

La implementación de un sistema de gestión de la calidad utiliza ciertamente muchos recursos adicionales en cualquier organización. ¿Cómo conciliar ambos procesos?

Fue precisamente esta pregunta el origen del cambio acelerado en el desempeño de la planta de la empresa A en Canadá y la razón del presente artículo.

\subsection{Consideraciones para la implementación de sistemas de gestión de la calidad}

Como se ha comentado al inicio del artículo, la exigencia de implementar un sistema de gestión de la calidad surge de la necesidad de proveer una respuesta predecible a los clientes.

La implementación de un sistema de gestión de la calidad basado en la norma ISO 9001 nace de una necesidad doble. Por una parte, la norma da las líneas de guía para construir un sistema que considere 
todos los puntos de control de nuestros procesos; y por otra parte, la obtención de un certificado ISO 9001 puede servir como una herramienta eficaz de márketing.

Sin embargo, es un error implementar un sistema basado en ISO 9001 solamente por el márketing o porque el mercado objetivo lo requiere.

Es necesario definir claramente de qué manera el proyecto se inscribe en el planeamiento estratégico general de la empresa, el objetivo particular del proyecto, los límites, los recursos a utilizar, las fechas y los medios de comunicación con los involucrados. Por recursos se entienden, aparte de las máquinas y el espacio físico, el tiempo necesario de todos los involucrados para elaborar los procedimientos.

En el caso de la empresa A Canadá el problema eran los recursos humanos y los medios de comunicación. Los recursos humanos ya estaban ocupados con la reorganización de las operaciones, y respecto a los medios de comunicación, quería evitarse el tener que llevar un control de copias físicas de documentos distribuidos. Nótese que aun cuando la decisión de adoptar la norma ISO 9001 tuvo un fuerte contenido de márketing e imagen, no se descuidaba el aspecto de implementarla correctamente, de forma que la certificación fuese sostenible en el tiempo.

\subsection{La implementación de la norma ISO 9001 como herramienta de mejora}

Ante la disyuntiva de utilizar los recursos para la reorganización de las operaciones o para la implementación correcta del sistema de gestión de la calidad, la dirección de la empresa A analizó la situación con la ayuda de un asesor externo. Desde un principio quedó en claro que el rol del asesor no es el de construir el sistema sino el de ayudar en el análisis y la elaboración de este; y fue así como surgió una idea bien acogida por ambas partes: era posible utilizar la norma ISO 9001, a la vez, como guía y como herramienta para continuar con la reorganización.

Este cambio de enfoque, en el que la norma ISO 9001 pasa de ser una guía para documentar los procesos existentes y mejorarlos ligeramente a ser una herramienta para continuar con la reorganización es bastante interesante, pero no es posible aplicarla en todos los casos. La experiencia en la empresa A Canadá sugiere que algunos de los requisitos son: 
- Tener una idea clara de hacia dónde se dirige la reorganización.

- El personal involucrado debe estar identificado con los cambios iniciados, y que estos estén dando buenos resultados.

La ausencia de estos requisitos retardaría seriamente la implementación de la norma, porque habría potencialmente cambios importantes por hacer, lo que obligaría a estar continuamente cambiando la documentación sobre los procesos.

\section{Implementación de la norma ISO 9001 en la empresa A}

\subsection{Análisis preliminar}

La implementación de la norma ISO 9001 en la empresa A Canadá se inició durante el primer trimestre del año 2007; sin embargo, desde el inicio del proceso de reorganización se habían identificado dos de las características más importantes para los clientes de la empresa A Canadá:

- La entrega a tiempo de los pedidos.

- Que los pedidos contengan exactamente lo que el criador solicita. Esto incluye las secuencias de identificadores y sus accesorios o herramientas.

Se había identificado entonces que la respuesta predecible que esperaban los usuarios finales estaba compuesta por un tiempo de entrega que respete los límites acordados y por el contenido exacto de las órdenes.

Con estos elementos se definió el documento más importante de un sistema de gestión de la calidad basado en la norma ISO 9001: la política de calidad, que fue utilizada por la empresa A Canadá como elemento de contacto entre:

- la necesidad del mercado objetivo,

- los recursos con los que debía contar la empresa para atender ese mercado,

- la definición de los procesos internos que utilizarían los recursos disponibles, y

- la interacción entre estos procesos. 
El resultado es un sistema en el que toda actividad, decisión, documento, registro y resultado están alineados con la política de calidad, de forma que las redundancias, las actividades que no agregan valor y la mejora continua son el resultado natural de la aplicación y puesta en marcha de dicho sistema.

Fue precisamente este enfoque el que permitió integrar, de forma natural, la implementación de la norma a las actividades de reorganización en curso, pues a partir del momento de la definición de la política de calidad, incluso antes de haber documentado los procedimientos o iniciado oficialmente las actividades de mejora continua, ya se disponía de la idea general y oficial de hacia dónde debían orientarse los cambios, así como los indicadores que reflejarían el resultado de estos cambios.

\subsection{Construir la documentación}

Este es probablemente uno de los puntos más conocidos de un sistema de gestión de la calidad basado en la norma ISO 9001. En la experiencia de la empresa A Canadá este punto fue también fuente de preocupación por la aparente enorme cantidad de documentos para redactar, revisar, mantener actualizados, distribuidos y conocidos por el personal que ejecuta los procesos.

Un análisis detallado de las necesidades y características de la organización arrojó un resultado tanto alentador como inesperado, que permitió construir rápidamente una documentación sólida. La importancia de este análisis radica en que se identifica la forma en que la norma debe ser implementada en la organización, de manera que los procesos no se adaptan a los procedimientos escritos, sino que los procedimientos escritos reflejan los procesos.

La política de calidad fue el primer documento establecido, el segundo fue el proceso general. Este fue concebido para el caso de la empresa A Canadá como un diagrama de bloques en el que se detallaban todos los procesos internos agrupados en familias o bloques de procesos, como en el ejemplo siguiente: 
Figura 1

Proceso general - Empresa A Canadá

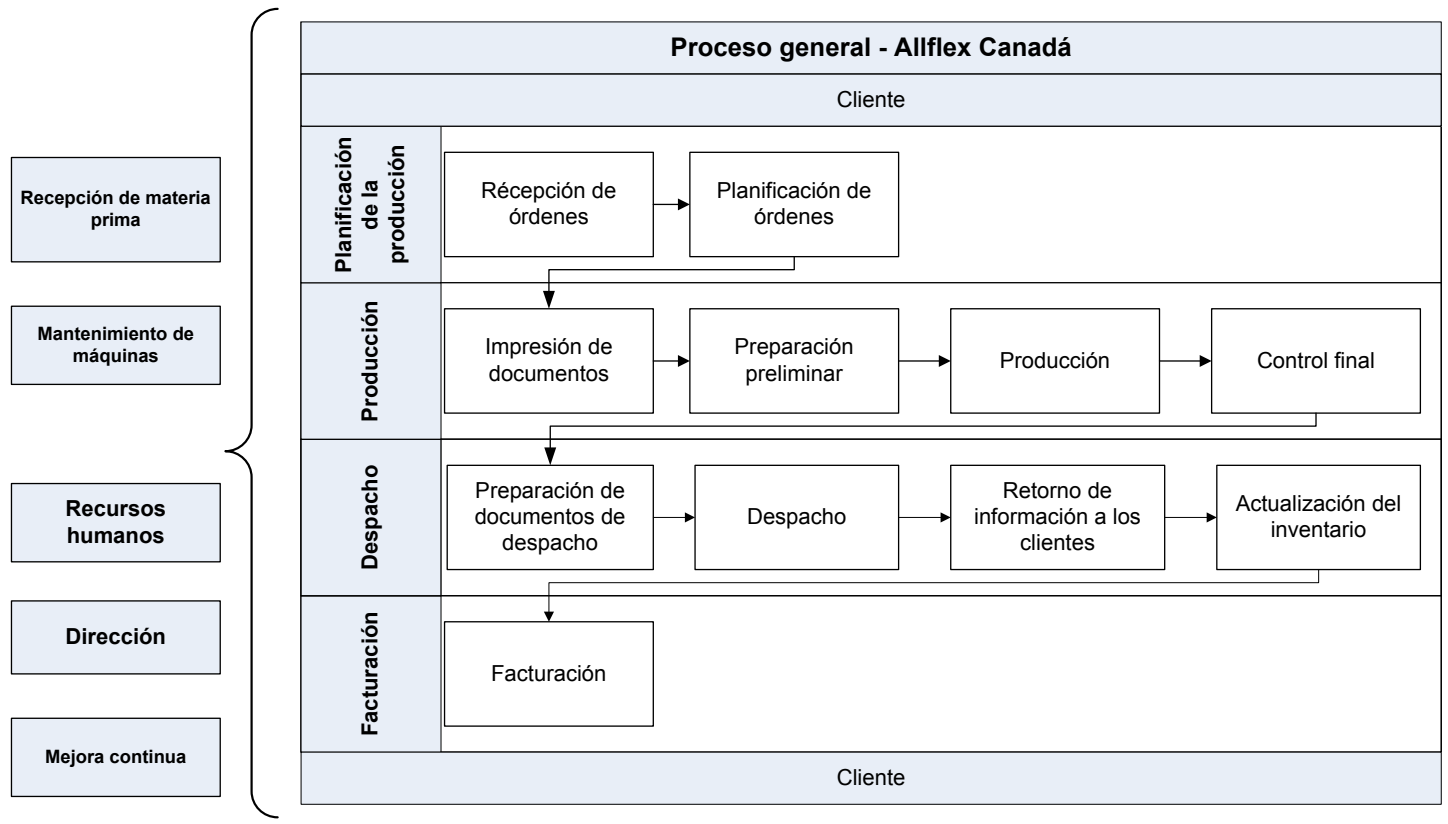

Fuente: Manual de calidad de la empresa A Canadá.

Elaboración propia.

Cada uno de los procesos indicados en este diagrama debe contar con su descripción documentada, que no es necesariamente una descripción escrita y detallada paso por paso de cómo ejecutar el proceso.

La empresa A Canadá descartó la posibilidad de utilizar solamente texto para la documentación de estos procesos y en su lugar optó por el uso de diagramas de flujo, por las siguientes razones:

- Como la documentación debía ser bilingüe, el uso exclusivo de texto significaría un mayor costo para el mantenimiento de dicha documentación.

- La descripción debía ser lo suficientemente flexible como para permitir una edición rápida que reflejara los cambios en los procedimientos originados por la reorganización en curso y por las mejoras futuras. 
- La descripción debía ser lo suficientemente visual, con el fin de que el entrenamiento del nuevo personal sea más fácil y en consecuencia más rápido.

El resultado fue que todos los procesos indicados tenían una representación, a la que se denominó Procedimiento, y que tiene el siguiente formato:

Figura 2

Ejemplo de procedimiento

\section{Proceso 1}

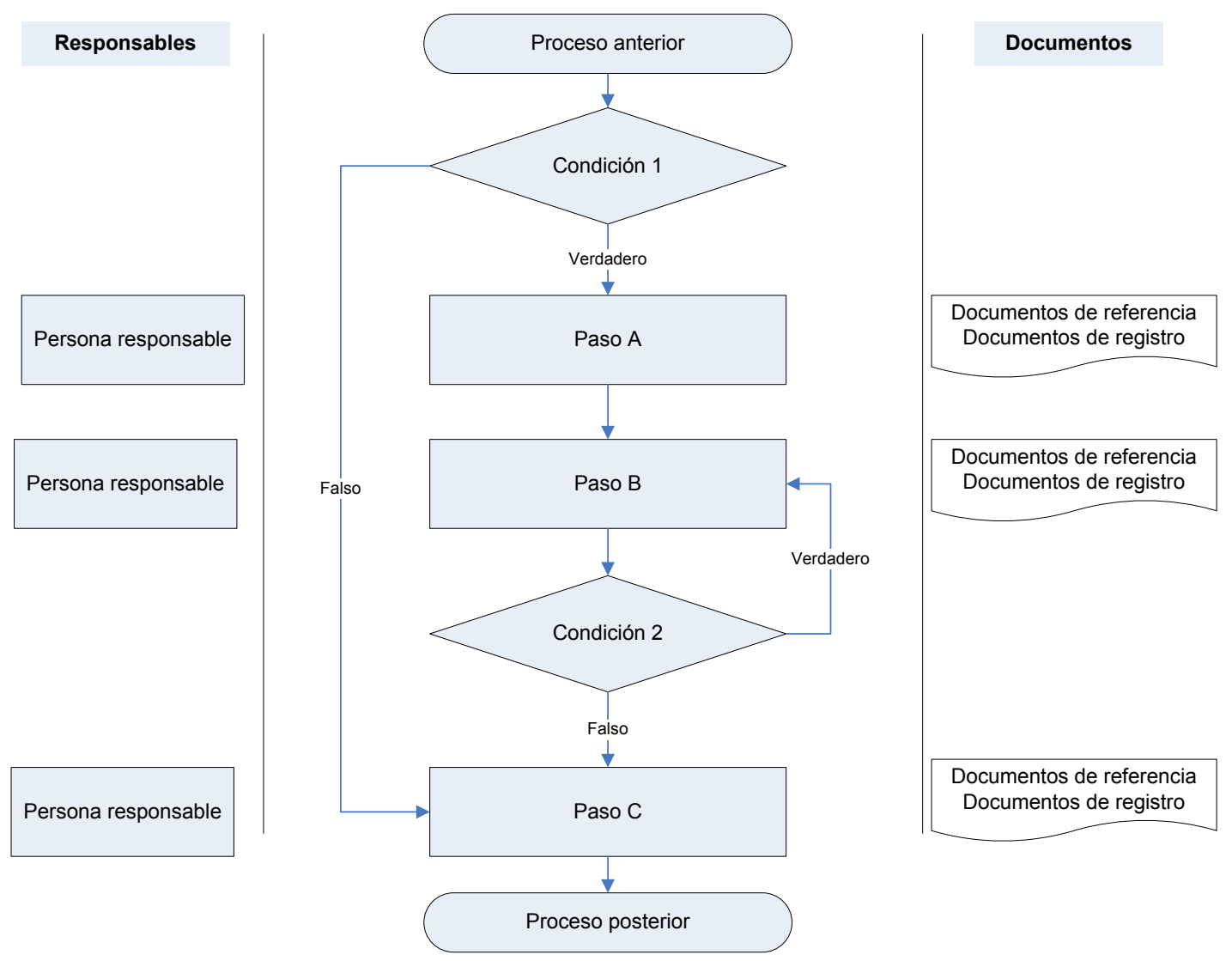

Fuente: Modelo de procedimiento de la empresa A Canadá.

Elaboración propia. 


\subsection{Diferencia entre procedimiento e instrucción de trabajo}

Sin embargo, por flexible que fueran estos diagramas para ilustrar los procedimientos, no permitían el nivel de detalle requerido para ciertos casos, en los que el uso de texto e imágenes es la mejor opción para eliminar toda duda de la persona responsable de ejecutar la tarea, e incluso para ayudar en el entrenamiento de personal nuevo o de reemplazo. Para esos casos se crearon documentos especiales, que en la documentación de la empresa A Canadá fueron denominados instrucciones de trabajo.

Una instrucción de trabajo es definida como un manual completo y detallado para aquellos pasos descritos en los procedimientos, que dado su impacto en el resultado final requerían un mayor grado de control en su ejecución. A modo de ilustración, en el diagrama de la figura 2 (Ejemplo de procedimiento) podría determinarse que solamente el paso $\mathrm{B}$ requería una instrucción de trabajo.

Esta forma de estructurar la documentación permitió un mejor uso de los limitados recursos disponibles, pues se cumplía con las exigencias de la norma al tener procedimientos claros y definidos, al mismo tiempo que se disponía de instrucciones de trabajo mucho más extensas y detalladas para aquellas actividades que lo requerían.

\subsection{La documentación debe reflejar lo que realmente se ejecuta}

El asesor externo advirtió a la empresa sobre la tendencia de algunas organizaciones a elaborar procedimientos e instrucciones de trabajo que ostentan una imagen ideal de los procesos internos pero que no reflejan la realidad. Esta tendencia es el resultado natural de querer presentar una documentación óptima para las auditorías con la finalidad de aumentar la probabilidad de obtener el certificado; sin embargo, esta misma tendencia tiene un efecto negativo a largo plazo por los siguientes motivos:

- Es equivalente a implementar la norma ISO 9001 solamente con fines de márketing o imagen.

- Es probable que en la auditoría de certificación la diferencia entre lo que el documento describe y lo que pasa en realidad genere observaciones o no conformidades que pueden dar como resultado la no obtención del certificado. 
- En caso de obtenerse el certificado esa misma diferencia puede aún generar observaciones o no conformidades en las auditorías de control posteriores, que podrían determinar la pérdida del certificado, con lo que todo el trabajo sería en vano.

Al tomar esto en consideración se decidió, con el objetivo de ahorrar tiempo en la redacción y garantizar la solidez de la documentación, utilizar las notas y análisis efectuados durante la reorganización de las actividades como base para la elaboración de los procedimientos. En efecto, al hacer esto se respetaban los cambios ya realizados y había una garantía de que los procedimientos reflejaran fielmente lo que el personal ejecutaba.

\subsection{Procesos ejecutados al exterior de la empresa A Canadá}

La norma ISO 9001 permite excluir ciertos elementos obligatorios bajo ciertas circunstancias. La empresa A Canadá se vio en la necesidad de aplicar estas exclusiones en razón de sus operaciones descentralizadas, en las que por políticas corporativas algunas plantas ejecutan procesos, tales como compras o tratamiento de información, para otras. La estructura de la documentación permitió identificar rápidamente estas exclusiones e incluso preparar la justificación necesaria, así como las medidas de contingencia en caso de que los procesos dejaran de ser ejecutados por el recurso externo o que la ejecución no se diese de acuerdo a los parámetros establecidos.

El hecho de que otras plantas de la organización contaran con el certificado ISO 9001 ayudó en gran medida para estos casos, pues aunque las actividades de la empresa A Canadá no estaban cubiertas por el certificado, gracias a estas plantas fue posible establecer los puntos de conexión entre los certificados.

\subsection{Indicadores de gestión}

Uno de los requisitos para la obtención del certificado ISO 9001 indica que la organización debe vigilar, medir y analizar sus procesos (Landry 2001, p.59), y la forma más adecuada para cumplir con este requisito es el uso de indicadores de gestión; sin embargo, el caso particular de la empresa A Canadá obligó a crear y justificar el uso de pocos indicadores de gestión. 
El análisis preliminar y el proceso general permitieron establecer que debido a la extensión de las tareas, la cantidad de personal involucrado y el tiempo requerido para la ejecución de los procesos habría sido un ejercicio inútil contar con indicadores para cada proceso. En consecuencia, aun cuando hubiera sido (y es) posible establecer indicadores para cada uno de los procesos, se decidió utilizar indicadores globales que estuvieran directamente relacionados con la performance económica de la empresa y las características de la respuesta predecible que los clientes esperaban.

De esta manera, se define uno de los indicadores de gestión al compilar la estadística de órdenes entregadas a tiempo. La orden entregada a tiempo consiste en que dado el acuerdo con un cliente en particular, para un artículo en particular, las órdenes recibidas para este son despachadas o no dentro del tiempo acordado. Nótese que el indicador no es cuánto tiempo toma despachar las órdenes sino qué porcentaje de sus órdenes son entregadas dentro de los límites establecidos por cada cliente.

El ejemplo y la evolución de este indicador se presentan en el punto 5.1 (Respuesta de los clientes).

\section{PUESTA EN MARCHA DEL SISTEMA DE GESTIÓN DE LA CALIDAD}

\subsection{Comunicación interna y entrenamiento del personal}

Una vez terminada la redacción de los procedimientos se estableció la forma de comunicárselos al personal y ofrecerle el entrenamiento necesario para que el sistema fuese puesto en marcha correctamente.

En el caso de la empresa A Canadá se aprovechó el hecho de que el mismo personal había participado en las mejoras iniciadas y que la documentación fue creada como consecuencia de las tareas ejecutadas. El resultado fue un tiempo de entrenamiento muy corto y una curva de aprendizaje bastante acelerada, pues lo que más tiempo tomó fue implantar el hábito de leer los procedimientos (todo proceso tiene un procedimiento) y las instrucciones de trabajo (las tareas críticas tienen instrucciones de trabajo) en lugar de preguntar a otros trabajadores sobre la forma de ejecutar las tareas.

Es importante mencionar que toda la comunicación interna y el entrenamiento fueron documentados, utilizando los ya existentes formatos y procedimientos. 


\subsection{Publicación de la documentación}

Para la publicación de la documentación se eligió la red interna de la empresa para los procedimientos. Esta forma de implementación eliminó la necesidad de mantener un registro de las versiones impresas de los diferentes procedimientos en la organización porque la versión publicada es siempre la más actualizada.

Para justificar esta forma de publicación se fijaron las siguientes exigencias para los documentos electrónicos:

- Los documentos publicados deberán ser de acceso universal y el contenido se adecuará a las exigencias usuales de la norma, tales como identificación de versión y de los cambios realizados desde la última versión.

- Será imposible modificar el contenido de los documentos una vez publicados. Esta disposición tiene por finalidad evitar que los procedimientos sean modificados voluntaria o involuntariamente entre el momento de la publicación y el momento en que son consultados por el personal.

- Debe guardarse un histórico de los cambios en los procedimientos, o en su defecto la versión completa de los procedimientos retirados o mejorados.

La empresa A Canadá determinó que la documentación sea publicada en el formato Adobe Acrobat en la red interna de la empresa.

\subsection{Recursos humanos}

La puesta en marcha del incipiente sistema y de los procedimientos de recursos humanos permitió identificar rápidamente una debilidad en la empresa, que era la no existencia de planes de contingencia formales para cuando ciertas personas clave de la organización no estuvieran presentes.

Se estableció entonces una política y un plan para el entrenamiento formal y documentado de personal de remplazo. Todas estas acciones fueron debidamente documentadas y sirven hasta el día de hoy de sustento para las auditorías. 


\subsection{Registros}

La norma y los propios procedimientos de empresa A Canadá exigen guardar un registro de la ejecución de las tareas y de acciones de prevención y corrección. En este caso se implementó para el registro de la realización del producto un sistema de doble registro:

- Durante la realización del producto se utilizan documentos impresos en papel, que son utilizados por el personal al momento como una de sus fuentes de información para ejecutar las tareas.

- La empresa A Canadá guarda automáticamente un registro informático de la ejecución de estas mismas tareas.

El registro informático existía desde antes y su uso fue reforzado al momento de implementar la norma ISO 9001, pues da un acceso mucho más rápido a la información, lo que a su vez permite:

- Establecer los indicadores de gestión de forma fiable, pues no se depende de formatos llenados por nadie sino de información capturada automáticamente.

- Ver el detalle de la ejecución de las tareas: quién hizo qué parte del proceso, qué elementos fueron incluidos en los paquetes enviados a los clientes.

- Extraer información que ayude en la respuesta a preguntas y quejas de los clientes, así como en el análisis para la mejora de los procesos.

Los documentos en papel son archivados por un tiempo determinado, luego de lo cual son destruidos tanto para liberar espacio de almacenamiento como para cumplir con la reglamentación del cuidado de la información confidencial de los clientes. Por el momento el registro informático guarda indefinidamente la información, pero se ha establecido también un tiempo luego del cual esta información puede ser eliminada de los servidores de la empresa. Este tiempo está determinado por las disposiciones legales a las cuales la empresa A Canadá y sus clientes están sujetos. 


\section{RESULTADOS}

\subsection{Respuesta de los clientes}

El análisis preliminar estableció que los clientes de la empresa $\mathrm{A} \mathrm{Ca-}$ nadá son sensibles a la entrega a tiempo de sus órdenes, de forma que la implementación de los cambios en la empresa fue orientada, entre otras cosas, a mejorar el porcentaje de órdenes entregadas a tiempo. La información y capacidad de control que la norma ISO 9001 dio a la empresa fue puesta también al servicio del mismo objetivo. En el gráfico siguiente se ve la evolución de este porcentaje:

\section{Gráfico 1}

Porcentaje de órdenes entregadas a tiempo

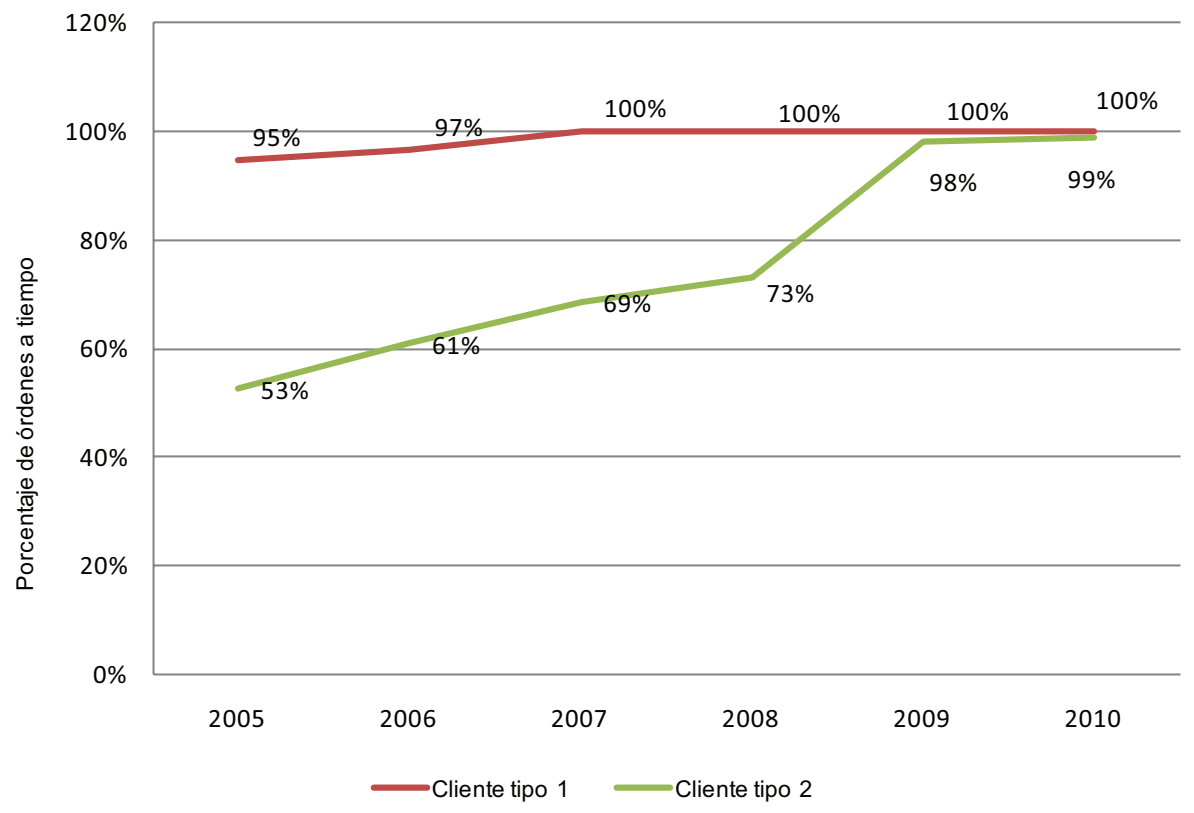

Fuente: Empresa A Canadá.

Elaboración propia.

Se observa que desde antes de la implementación de la norma ISO 9001, en el 2007, había una mejora en este indicador; y al mismo tiempo puede verse que luego de haber completado la implementación hay una mejora acelerada en este. Este cambio fue notado paulatinamente por los clientes. 
Un efecto favorable no previsto por este cambio fue la disminución de casos especiales o de órdenes declaradas urgentes con la única finalidad de recibirlas a tiempo. Dado que el tiempo de respuesta se tornó mucho más predecible y estable ya no era necesario enviar órdenes aparte para casos de urgencia, pues se sabía que los clientes recibirían sus órdenes dentro de los plazos establecidos con el solo hecho de transmitir dichas órdenes.

Otro punto bien recibido por los clientes fue la mejora en la calidad y el contenido de las respuestas a las preguntas y quejas. En efecto, con un acceso mejorado a la información exacta del historial de cada orden y al número de rastreo de entrega fue posible disipar dudas en cuanto al contenido de los paquetes enviados así como de su fecha final de entrega; de la misma forma era ahora posible identificar las causas reales del error e implementar medidas correctivas.

Adicionalmente, y siempre dentro del marco de la norma ISO 9001, la encuesta obligatoria a los clientes permitió tener incluso más detalle sobre los puntos que fueron percibidos como mejoras notables y sobre los puntos en los que los clientes mismos esperan mejoras. Esta información, junto con la proveniente de análisis internos, empezó a utilizarse para dirigir los planes de mejora continua exigidos por la norma.

\subsection{Respuesta del Grupo A}

La mejora en la respuesta de los clientes ocasionó también que la empresa A revisara ciertas decisiones que habían tenido como consecuencia que parte de la producción destinada al mercado canadiense fuese procesada por otras plantas del grupo.

En forma ordenada y progresiva, la planta de la empresa A Canadá recibió nuevamente la carga de trabajo generada por los clientes del mercado canadiense.

La producción de identificadores oficiales producidos por A Canadá tiene la evolución siguiente: 
Gráfico 2

Comparativo de cantidad de identificadores oficiales producidos por la empresa A Canadá

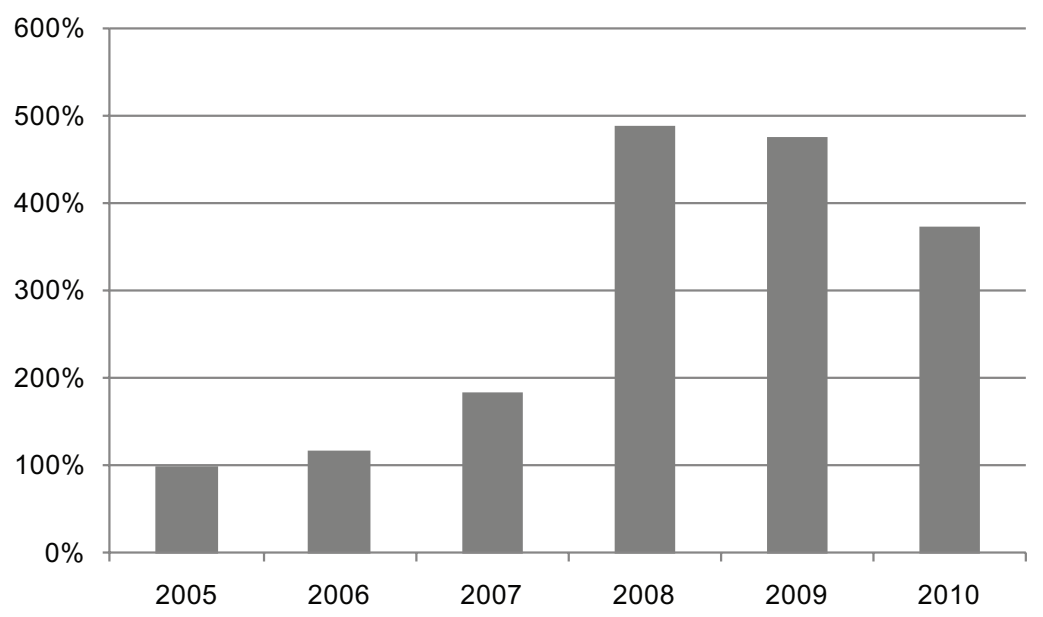

Fuente: Empresa A Canadá.

Elaboración propia.

En el gráfico 2 se establece la cantidad producida en el año 2005 como base para comparar los años siguientes. Es claro que la decisión de traer nuevamente la producción para el mercado canadiense a la planta de la empresa A Canadá significa una mejora en la logística del grupo, pero no pudo ser efectuada sin un sistema que soportase adecuadamente esta producción.

\subsection{Mejora continua}

El aumento en la producción local trajo a su vez inversiones y otras oportunidades de mejora en el funcionamiento de la planta, iniciándose de esta forma un círculo virtuoso de mejora continua.

Por otro lado, es claro para el personal de la planta de A Canadá que estas mejoras son resultado del esfuerzo del mismo personal en la puesta en práctica del sistema de gestión de calidad y en identificar posibilidades de mejora en los procesos con la finalidad de implementar ordenadamente estas mejoras. 


\section{CONCLUSIONES Y RECOMENDACIONES}

- Como toda decisión estratégica, la decisión de implementar un sistema de gestión de la calidad basado en la norma ISO 9001 debe ser parte de una estrategia de mayor envergadura y estar acompañada del apoyo de la alta dirección de la empresa. En contrapartida, es responsabilidad de la organización cumplir con la ejecución de los procedimientos establecidos y buscar e identificar las oportunidades de mejora continua.

- La implementación de un sistema de gestión de la calidad basado en la norma ISO 9001 no es incompatible con un proceso preexistente de reorganización de las actividades de la empresa, e incluso puede ser utilizado como herramienta para mejorar el control de los cambios y reducir el tiempo de la implementación de estos.

- El análisis preliminar de la situación y características de la empresa, así como de su mercado, son los elementos clave para una implementación correcta de la norma ISO 9001.

- El sistema de gestión de calidad debe estar basado en la idea de reflejar en documentos las tareas que son ejecutadas en la empresa. En el mismo sentido, debe evitarse el implementar dicho sistema únicamente con fines de márketing o de mejora de la imagen en el mercado.

- El uso de documentación y registros electrónicos es recomendable porque permite un mejor y más rápido acceso a la información relevante en función de la necesidad que se presente: compilar estadísticas, revisar detalles en la ejecución de las tareas, analizar la pertinencia o no de cambios en los procedimientos. Adicionalmente, esta documentación electrónica garantiza que la versión que el personal consulta es siempre la más actualizada, eliminando así la necesidad de guardar un registro detallado de las versiones impresas de procedimientos y su ubicación en la organización (quién tiene qué versión). 


\section{REFERENCIAS}

1. Landry, Pierre D. (2001). Guide ISO 9000:2000 Lignes directrices pour l'application des normes ISO 9000. (3a. ed.). Montreal: l'Association Canadienne de Normalisation.

2. Rahman, A. H., Wan, A. I. \& Mohd, I. H. (2005). ISO 9001:2000 Certification: A marketing tool. Recuperado el 8 de marzo de 2011, de http://www.lib.usm.my/elmuequip/conference/Documents/ICOL\%20 2005\%20Paper\%208\%20Habsah,\%20Abdul\%20Akla\%20\&\%20 Mohd\%20Idris.pdf. 